\title{
Quoi de neuf dans le Guide canadien d'immunisation (de novembre 2016 à novembre 2018)?
}

\author{
A Fleurant-Ceelen ${ }^{1}$, M Tunis ${ }^{1}$, A House ${ }^{1}$, au nom du Comité consultatif national de l'immunisation \\ $(\mathrm{CCNI})^{*}$
}

\section{Résumé}

Le Guide canadien d'immunisation est une ressource en ligne présentant aux fournisseurs de soins de santé et aux professionnels de la santé publique du Canada des recommandations fondées sur des données probantes relativement à l'utilisation des vaccins et aux méthodes $d^{\prime}$ administration des vaccins. Son contenu est fondé sur les plus récentes recommandations du Comité consultatif national de l'immunisation (CCNI) et du Comité consultatif de la médecine tropicale et de la médecine des voyages (CCMTMV). Le Guide canadien d'immunisation (GCl) est fréquemment actualisé en ligne afin de tenir compte de nouvelles données probantes et de modifications aux indications de produits. Entre novembre 2016 et novembre 2018, de nouvelles recommandations ont été ajoutées au Guide, ou des recommandations y ont été actualisées. Ces nouveautés concernent notamment les chapitres sur les Méthodes d'administration des vaccins et l'Immunisation des sujets immunodéprimés, I'Immunisation durant la grossesse et l'allaitement, ainsi que sept vaccins actifs (le choléra, la diarrhée du voyageur, la grippe, I'hépatite $A$, I'hépatite $B$, I'herpès zoster, le virus du papillome humain et la coqueluche). Il comprend aussi des mises à jour récentes sur la prophylaxie post-exposition contre la rougeole.

\section{Affiliation}

${ }^{1}$ Centre de l'immunisation et des maladies respiratoires infectieuses, Agence de la santé publique du Canada, Ottawa (Ontario)

\section{*Correspondance:}

phac.naci-ccni.aspc@canada.ca

Citation proposée : Fleurant-Ceelen A, Tunis M, House A, au nom du Comité consultatif national de I'immunisation (CCNI). Quoi de neuf dans le Guide canadien d'immunisation (de novembre 2016 à novembre 2018)?, Relevé des maladies transmissibles au Canada 2018;44(12):398-403. https://doi.org/10.14745/ccdr. v44i12a06f

Mots clés: Immunisation, CCNI, Canada, mise à jour, vaccin

\section{Introduction}

Depuis 1964, le Comité consultatif national de l'immunisation (CCNI) prodigue des conseils sur les vaccins aux gouvernements et aux professionnels, aussi bien au Canada qu'à l'étranger. Pour ce faire, il distribue une série de produits d'information répondant aux besoins d'une variété d'auditoires. Le CCNI met au point des produits techniques détaillés, par exemple des analyses documentaires ou des déclarations, à l'intention des experts en immunisation et des décideurs. Le CCNI élabore aussi des produits sommatifs et translationnels, notamment des sommaires de déclarations publiés dans le Relevé des maladies transmissibles au Canada, ainsi que des mises à jour dans le Guide canadien d'immunisation (GCl). Ces produits sont destinés aux professionnels de première ligne de la santé publique et des soins cliniques. La figure 1 donne un aperçu du processus de production du CCNI.
Figure 1 : Comité consultatif national sur l'immunisation - Processus de production

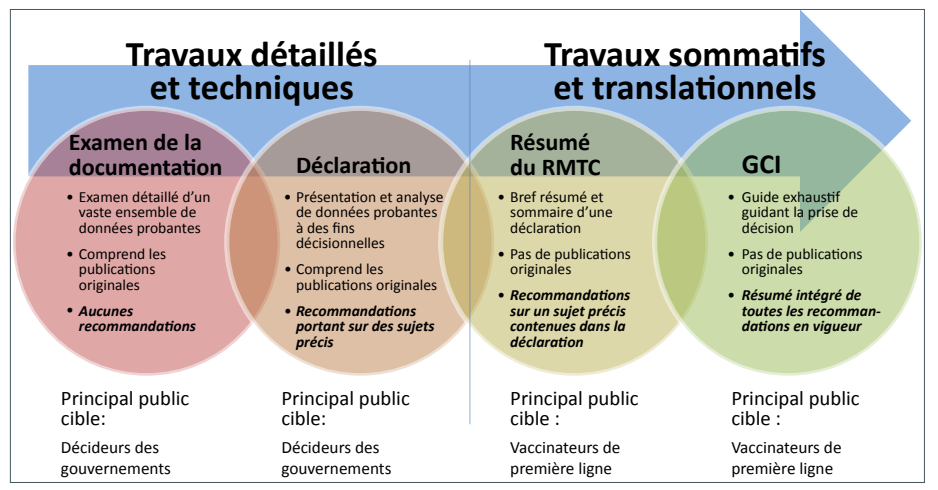

Abréviations $\mathrm{GCl}$, Guide canadien d'immunisation; RMTC, Relevé des maladies transmissibles au Canada 
Depuis 1979, le $\mathrm{GCl}$ renseigne les dispensateurs de vaccins de première ligne sur les mesures cliniques à prendre en matière d'immunisation (2). Depuis 2012, le GCl est devenu un document évolutif offert en ligne (3). Il est régulièrement mis à jour au fur et à mesure que le $\mathrm{CCNI}$ formule de nouvelles recommandations. Il comprend également des recommandations formulées par le Comité consultatif de la médecine tropicale et de la médecine des voyages (CCMTMV) relativement aux vaccins et à des questions connexes. Le $\mathrm{GCl}$ ne traite pas de questions économiques ou sociales liées à l'immunisation. Toutefois, il met en évidence l'évolution de l'épidémiologie des maladies, les indices de problèmes liés à l'innocuité et les difficultés en matière d'approvisionnement de vaccins.

Le $\mathrm{GCl}$ est divisé en cinq parties : Information clé sur I'immunisation; Innocuité des vaccins; Vaccination de populations particulières; Vaccins actifs; et Agents d'immunisation passive.

La présente mise à jour vise à donner un aperçu des modifications apportées au $\mathrm{GCl}$ entre novembre 2016 et novembre 2018. Ces modifications touchent de l'information clé sur l'immunisation, la vaccination de populations particulières, les vaccins actifs et la prophylaxie post-exposition contre la rougeole.

\section{Information clé sur l'immunisation}

Le chapitre sur les Méthodes d'administration des vaccins (4) a été mis à jour. II comprend maintenant des Lignes directrices pour le choix de l'aiguille mettant l'accent sur l'importance de choisir la longueur de l'aiguille selon chaque cas pour une injection intramusculaire, et d'évaluer le niveau de viscosité de l'agent immunisant, ainsi que l'âge, le poids et la masse musculaire du bénéficiaire. L'utilisation d'aiguilles à filtre n'est pas recommandée, car des ingrédients actifs comme les adjuvants pourraient être filtrés pendant l'injection. On y note qu'une injection peut être pratiquée sur un tatouage ou une marque de naissance superficielle, mais qu'il faut éviter les endroits où le système lymphatique pourrait être perturbé. Ce chapitre comprend aussi un nouveau tableau présentant des stratégies de gestion de la douleur liée à la vaccination, destinées à des patients de tout âge. Au sujet de la combinaison du contenu des flacons multidoses, on conseille aux fournisseurs de soins de santé de s'en tenir aux politiques et procédures de la province ou du territoire, ou de l'organisation.

\section{Vaccination de populations particulières}

Deux chapitres ont fait l'objet de mises à jour : Immunisation des sujets immunodéprimés et Immunisation durant la grossesse et l'allaitement.

Le chapitre consacré à l'Immunisation des sujets immunodéprimés (5) comprend maintenant des tableaux présentant des recommandations sur l'immunisation par vaccin, en tenant compte de l'immunodéficience primaire et de I'immunodéficience acquise (secondaire), des candidats à une transplantation ou des receveurs d'une transplantation et des personnes infectées par le VIH. On y trouvera de l'information inédite sur les déficiences de l'immunité naturelle, les facteurs à prendre en considération pour les vaccins contre la rougeole, les oreillons et la rubéole, ainsi que la varicelle chez les sujets qui présentent des déficits partiels en lymphocytes $\mathrm{T}$; des contre-indications en matière $d$ 'administration de vaccins viraux vivants pour certains cas de déficit en cellule phagocytaires et de traitement immunosuppresseur.

Le chapitre Immunisation durant la grossesse et I'allaitement (6) a été mis à jour pour tenir compte d'une nouvelle recommandation préconisant l'administration du vaccin contre la coqueluche entre la $27^{\mathrm{e}}$ et la $32^{\mathrm{e}}$ semaine de grossesse. II y est clairement dit que les vaccins contenant du thimérosal sont sûrs pendant la grossesse et devraient être administrés si c'est indiqué. L'administration d'immunoglobulines anti-Rh et d'autres produits sanguins, et l'administration d'une série de vaccins (antiméningococcique conjugué quadrivalent; antiméningococcique $B$; fièvre jaune; encéphalite japonaise) pendant la grossesse font également l'objet de nouvelles considérations.

\section{Vaccins actifs et immunisation passive}

Sept chapitres consacrés à des vaccins actifs ont été mis à jour, et l'information concernant la prophylaxie post-exposition contre la rougeole à l'aide de produits à base d'immunoglobuline a été actualisée.

\section{Choléra et Escherichia coli entérotoxinogène (diarrhée du voyageur)}

Les avantages de ce vaccin étant limités, le vaccin oral contre le choléra ne fait plus partie des recommandations courantes pour prévenir la diarrhée du voyageur. Le CCMTMV note qu'il peut être conseillé pour les personnes présentant un risque particulièrement élevé d'infection, de complications de santé ou qui risquent d'être fortement incommodées par la diarrhée du voyageur, par exemple les travailleurs humanitaires, les professionnels de la santé travaillant dans des pays endémiques, les voyageurs courant un risque élevé d'être exposés à de l'eau ou à de la nourriture contaminée, les sujets immunodéprimés et ceux atteints d'une maladie chronique qui courent un plus grand risque de souffrir des conséquences graves de la diarrhée du voyageur. De plus, le CCMTMV recommande que toutes les autres personnes prennent les mesures d'hygiène courantes (hygiène des mains, précautions relatives aux aliments et aux boissons) et se procurent les médicaments en vente libre pour la gestion de la diarrhée du voyageur $(7,8)$.

\section{Grippe}

Les recommandations relatives au vaccin contre la grippe saisonnière sont mises à jour chaque année en prévision de la grippe saisonnière (9).

\section{Hépatite A}

La posologie recommandée pour l'administration intramusculaire d'immunoglobulines (Ig IM) en prophylaxie pré-exposition 
et post-exposition pour l'hépatite A a été augmentée conformément à de nouvelles indications dans la monographie de produit.

\section{Hépatite B}

En s'appuyant sur les données relatives à l'immunogénicité et I'innocuité du vaccin contre l'hépatite $B$, le $C C N I$ a révisé sa recommandation relative à la dose de Recombivax $\mathrm{HB}^{\mathrm{MD}}$. Pour les nourrissons (nés de mères non infectées par le virus de I'hépatite $B$ ) et les enfants jusqu'à 11 ans, la dose est passée de $0,25 \mathrm{ml}$ à $0,5 \mathrm{ml}$. La dose de $0,25 \mathrm{ml}$ est jugée adéquate pour les enfants qui sont déjà immunisés et il n'est donc pas nécessaire de la répéter. Après la vaccination de personnes dont le système immunitaire est affaibli, on peut envisager une surveillance annuelle des niveaux d'anticorps contre le virus de I'hépatite B (11).

\section{Herpès zoster (zona)}

Le nouveau vaccin sous-unitaire recombinant (VRZ) ayant été homologué au Canada (Shingrix ${ }^{\mathrm{MD}}$ ), le CCNI recommande maintenant d'offrir ce vaccin aux personnes de 50 ans et plus qui ne présentent pas de contre-indication, y compris à celles qui ont reçu le WVCZ ou vaccin à virus vivant contre le zona (Zostavax ${ }^{\mathrm{MD}}$ ), à condition que ce dernier ait été administré au moins un an auparavant. Le CCNI recommande d'offrir deux doses du VRZ aux personnes qui ne présentent pas de contre-indication et qui ont connu un épisode de zona, et ce, au moins un an après le dernier épisode. Dans les cas où le VRZ est contre-indiqué, s'il est impossible à obtenir ou qu'il est trop cher, on peut envisager l'administration du VVVCZ aux personnes d'au moins 50 ans qui sont immunocompétentes et qui ne présentent pas de contre-indication. Le VRZ (mais pas le VVVCZ) peut être envisagé au cas par cas pour les adultes de 50 ans et plus dont le système immunitaire est affaibli (12-14). Les lignes directrices se sont enrichies de deux tableaux résumant les facteurs à prendre en considération pour choisir le vaccin contre le zona et pour I'administrer (12).

\section{Papillome humain}

Le vaccin contre le virus du papillome humain (VPH), VPH9, est maintenant recommandé pour les garçons et les filles immunocompétents de 9 à 14 ans selon un calendrier de vaccination à deux ou trois doses. Cependant, le CCNI continue de recommander que le vaccin soit offert aux jeunes femmes et jeunes hommes de 15 à 26 ans selon un calendrier à trois doses, de même qu'aux personnes de plus de 26 ans susceptibles d'être exposées au virus de manière continue. Cette recommandation est la même pour les vaccins VPH2 (pour les filles seulement) et VPH4. Le calendrier de vaccination contre le VPH (VPH2, VPH4 ou VPH9) à deux ou trois doses doit toujours prévoir au moins 24 semaines entre la première et la dernière dose. Les sujets immunodéprimés devraient toujours être immunisés selon un calendrier à trois doses prévoyant au moins 24 semaines entre la première et la dernière dose (15).

\section{Coqueluche}

Selon des données récentes, il serait possible de protéger efficacement les nourrissons contre la coqueluche en administrant le vaccin anticoquelucheux acellulaire (dcaT) pendant la grossesse. Il est maintenant recommandé $\mathrm{d}^{\prime}$ administrer ce vaccin entre la $27^{\mathrm{e}}$ et la $32^{\mathrm{e}}$ semaine de grossesse. $\mathrm{Si}$, pour une raison ou une autre, le vaccin ne peut pas être administré pendant cette période, il est possible d'offrir le dcaT à n'importe quel moment entre la $13^{\mathrm{e}}$ semaine de grossesse et l'accouchement (16).

\section{Rougeole}

De nouvelles données probantes indiquent que la dose d'immunoglobulines $(\mathrm{lg})$ recommandée auparavant n'offre plus une protection optimale pour la prophylaxie post-exposition contre la rougeole. Le CCNI a mis à jour comme suit ses recommandations relatives à la posologie, aux indications et aux voies d'administration d'immunoglobulines humaines contre la rougeole en PPE (17) :

- Les sujets immunocompétents de six mois et plus qui ont été exposés au virus de la rougeole et qui ne présentent pas de contre-indication devraient recevoir le vaccin contre la rougeole, la rubéole et les oreillons dans les 72 heures qui suivent l'exposition

- Si le volume d'injection n'est pas une préoccupation importante, les nourrissons de moins de six mois devraient recevoir les lg IM à raison de 0,5 mL/ $\mathrm{kg}$, jusqu'à une dose maximale de $15 \mathrm{~mL}$ administrée sur plusieurs sites d'injection

- $\quad$ Si le volume d'injection n'est pas une préoccupation importante, les nourrissons de six à 12 mois, dont l'exposition à la rougeole est décelée après 72 heures et dans les six jours suivants devraient recevoir des immunoglobulines par voie intramusculaire (à raison de $0,5 \mathrm{~mL} / \mathrm{kg}$ ), jusqu'à une dose maximale de $15 \mathrm{~mL}$ administrée sur plusieurs sites d'injection

- $\quad$ Si le volume d'injection n'est pas une préoccupation importante, les personnes susceptibles qui sont enceintes ou immunodéprimées peuvent recevoir des Ig IM à raison de $0,5 \mathrm{~mL} / \mathrm{kg}$, tout en tenant compte du fait que les personnes de $30 \mathrm{~kg}$ ou plus ne recevront pas les concentrations d'anticorps contre la rougeole qui sont jugées nécessaires pour offrir une protection complète

- Lorsque le volume d'injection est une préoccupation importante ou chez les personnes de $30 \mathrm{~kg}$ ou plus, l'immunoglobuline peut être administrée par voie intraveineuse (lg IV) à une dose de 400 mg/kg (17) 
- Le CCNI ne recommande pas la PPE pour l'administration $d^{\prime}$ Ig contre la rougeole chez les personnes réceptives et immunocompétentes de plus de 12 mois en raison du faible risque de complications liées à la maladie et des défis cliniques liés à l'administration pour la prise en charge des cas et des contacts

Un résumé des modifications apportées aux recommandations touchant les vaccins actifs est présenté dans le tableau 1.

\section{Tableau 1 : Résumé des mises à jour concernant les vaccins actifs, novembre 2016 à novembre 2018}

\begin{tabular}{|c|c|c|}
\hline $\begin{array}{c}\text { Maladies } \\
\text { évitables par la } \\
\text { vaccination }\end{array}$ & $\begin{array}{l}\text { Recommandation } \\
\text { antérieure }\end{array}$ & Nouvelle recommandation \\
\hline $\begin{array}{l}\text { Choléra et } \\
\text { diarrhée du } \\
\text { voyageur }\end{array}$ & $\begin{array}{l}\text { Pas recommandé } \\
\text { systématiquement pour } \\
\text { les voyageurs }\end{array}$ & $\begin{array}{l}\text { Peut être envisagé pour des } \\
\text { voyageurs qui présentent un } \\
\text { risque élevé d'infection, de } \\
\text { complications ou qui risquent } \\
\text { d'être fortement incommodés }\end{array}$ \\
\hline Grippe & \multicolumn{2}{|c|}{$\begin{array}{l}\text { De nouvelles recommandations sont formulées chaque } \\
\text { année en prévision de la prochaine grippe saisonnière }\end{array}$} \\
\hline Hépatite A & $\begin{array}{l}\text { Pour une protection de } \\
\text { moins de trois mois, la } \\
\text { dose d'immoglobuline } \\
\text { administrée par voie } \\
\text { intramusculaire est de } \\
0,02 \mathrm{~mL} / \mathrm{kg} \text { de poids } \\
\text { corporel } \\
\text { Pour une protection de } \\
3 \text { mois ou plus, il faut } \\
\text { administrer } 0,06 \mathrm{~mL} / \\
\text { kg de poids corporel } \\
\text { et répéter tous les six } \\
\text { mois }\end{array}$ & $\begin{array}{l}\text { La dose normale } \\
\text { d'immoglobuline administrée } \\
\text { par voie intramusculaire est } \\
\text { de } 0,1 \mathrm{~mL} / \mathrm{kg} \text { pour les cas } \\
\text { de contacts avec le virus de } \\
\text { l'hépatite A en milieu familial } \\
\text { ou institutionnel. } \\
\text { Pour les personnes se rendant } \\
\text { dans des zones à risque élevé, } \\
\text { les doses prophylactiques sont } \\
\text { comme suit : } \\
\text { - Voyage d'un mois }=0,1 \mathrm{~mL} / \\
\text { kg } \\
\text { - Deux mois ou plus }=0,2 \\
\mathrm{~mL} / \mathrm{kg} \\
\text { - Répéter la dose de } 0,2 \mathrm{~mL} / \\
\mathrm{kg} \text { tous les deux mois }\end{array}$ \\
\hline Hépatite B & $\begin{array}{l}\text { Dose de Recombivax } \\
\mathrm{HB}^{\mathrm{MD}} \text { pour les enfants } \\
\text { entre } 0 \text { et } 10 \text { ans } \\
\text { (nés de mères non } \\
\text { infectées par le virus de } \\
\text { l'hépatite } \mathrm{B} \text { ) : } 0,25 \mathrm{~mL}\end{array}$ & $\begin{array}{l}\text { Il est recommandé } \\
\text { d'augmenter à 0,5 } \mathrm{mL} \text { la dose } \\
\text { de Recombivax HB }\end{array}$ \\
\hline Zona & $\begin{array}{l}\text { VRZ (Zostavax }{ }^{\mathrm{MD}} \text { ) est } \\
\text { recommandé pour } \\
\text { les adultes de } 50 \text { ans } \\
\text { et plus ne présentant } \\
\text { aucune contre- } \\
\text { indication }\end{array}$ & $\begin{array}{l}\text { - Le VRZ (ShingrixMD) est } \\
\text { recommandé pour les } \\
\text { adultes de } 50 \text { ans et plus ne } \\
\text { présentant aucune contre- } \\
\text { indication, y compris pour les } \\
\text { personnes ayant déjà reçu le } \\
\text { WVCZ. Cependant, ce dernier } \\
\text { doit avoir été administré au } \\
\text { moins un an auparavant. } \\
\text { - Dans les cas où le VRZ } \\
\text { est contre-indiqué, s'il est } \\
\text { impossible à obtenir ou } \\
\text { qu'il est trop cher, on peut } \\
\text { envisager l'administration } \\
\text { du WVVZ aux personnes } \\
\text { d'au moins } 50 \text { ans qui sont } \\
\text { immunocompétentes et qui } \\
\text { ne présentent pas de contre- } \\
\text { indication. }\end{array}$ \\
\hline
\end{tabular}

Tableau 1 (suite) : Résumé des mises à jour concernant les vaccins actifs, novembre 2016 à novembre 2018

\begin{tabular}{|c|c|c|}
\hline $\begin{array}{c}\text { Maladies } \\
\text { évitables par la } \\
\text { vaccination }\end{array}$ & $\begin{array}{l}\text { Recommandation } \\
\text { antérieure }\end{array}$ & Nouvelle recommandation \\
\hline Zona (suite) & $\begin{array}{l}\text { VRZ (Zostavax }{ }^{\mathrm{MD}} \text { ) est } \\
\text { recommandé pour } \\
\text { les adultes de } 50 \text { ans } \\
\text { et plus ne présentant } \\
\text { aucune contre- } \\
\text { indication }\end{array}$ & $\begin{array}{l}\text { - Le VRZ (mais pas le WVCZ) } \\
\text { peut être envisagé au cas par } \\
\text { cas pour les adultes de } 50 \\
\text { ans et plus dont le système } \\
\text { immunitaire est affaibli (12-14). }\end{array}$ \\
\hline $\begin{array}{l}\text { Papillome } \\
\text { humain }\end{array}$ & $\begin{array}{l}\text { Le vaccin VPH9 } \\
\text { est recommandé } \\
\text { selon un calendrier } \\
\text { d'immunisation en } \\
\text { trois doses, alors que } \\
\text { les vaccins VPH2 et } \\
\text { VPH4 doivent être } \\
\text { administrés selon un } \\
\text { calendrier en deux } \\
\text { ou trois doses chez } \\
\text { certains groupes de la } \\
\text { population. }\end{array}$ & $\begin{array}{l}\text { Pour certains groupes de la } \\
\text { population, il est maintenant } \\
\text { recommandé d'administrer } \\
\text { le vaccin VPH9 selon un } \\
\text { calendrier en deux ou trois } \\
\text { doses, de manière similaire aux } \\
\text { vaccins VPH2 et VPH4. }\end{array}$ \\
\hline $\begin{array}{l}\text { Coqueluche } \\
\text { (toux } \\
\text { coquelucheuse) }\end{array}$ & $\begin{array}{l}\text { Le vaccin dcaT } \\
\text { devrait être offert aux } \\
\text { femmes enceintes } \\
\text { pendant une éclosion } \\
\text { de coqueluche. }\end{array}$ & $\begin{array}{l}\text { Pour protéger les nourrissons, } \\
\text { le vaccin dcaT devrait être } \\
\text { offert à chaque grossesse, } \\
\text { idéalement entre la } 27^{e} \text { et la } \\
32^{\mathrm{e}} \text { semaine de gestation. }\end{array}$ \\
\hline Rougeole & $\begin{array}{l}\text { Posologie: } \\
\text { lorsqu'indiqué, } \\
\text { administrer une dose } \\
\text { d'immunoglobulines } \\
\text { de } 0,25 \mathrm{~mL} / \mathrm{kg} \text { par } \\
\text { voie intramusculaire } \\
\text { ou de } 0,5 \mathrm{~mL} / \\
\text { kg pour les sujets } \\
\text { immunodéprimés. } \\
\text { Populations : } \\
\text { Immunoglobulines par } \\
\text { voie intramusculaire } \\
\text { aux personnes } \\
\text { fragilisées de tout } \\
\text { âge qui se présentent } \\
\text { entre } 72 \text { heures } \\
\text { et six jours après } \\
\text { l'exposition; et aux } \\
\text { nourrissons de moins } \\
\text { de six mois, aux } \\
\text { femmes enceintes, } \\
\text { ou aux sujets } \\
\text { immunodéprimés qui } \\
\text { ont été exposés au } \\
\text { cours des six derniers } \\
\text { jours tout au plus. }\end{array}$ & $\begin{array}{l}\text { - La dose d'immunoglobines } \\
\text { administrée par voie } \\
\text { intramusculaire a été } \\
\text { augmentée à } 0,5 \mathrm{~mL} / \mathrm{kg} \text {, } \\
\text { jusqu'à un maximum de } 15 \mathrm{~mL} \\
\text { lorsque le volume d'injection } \\
\text { n'est pas une préoccupation. } \\
\text { - Voie d'administration : } \\
\text { immunoglobulines par } \\
\text { voie intraveineuse à raison } \\
\text { de } 400 \text { mg/kg lorsque le } \\
\text { volume d'injection est une } \\
\text { préoccupation importante ou } \\
\text { pour les sujets de } 30 \text { kg ou } \\
\text { plus. } \\
\text { - Modification des } \\
\text { populations proposées : le } \\
\text { CCNI ne recommande plus } \\
\text { la PPE pour l'administration } \\
\text { d'immunoglobulines } \\
\text { contre la rougeole chez les } \\
\text { personnes fragilisées et } \\
\text { immunocompétentes de } \\
\text { plus de } 12 \text { mois en raison du } \\
\text { faible risque de complications } \\
\text { liées à la maladie et aux défis } \\
\text { cliniques liés à l'administration } \\
\text { pour la prise en charge des cas } \\
\text { et des contacts. }\end{array}$ \\
\hline
\end{tabular}

Abréviations : CCMTMV, Comité consultatif de la médecine tropicale et de la médecine des voyages; CCNI, Comité consultatif national de l'immunisation; dcaT, diphtérie-coqueluchetétanos; Ig immunoglobuline; Ig IM, immunoglobine par voie intramusculaire; IV Ig, immunoglobine par voie intraveineuse; PPE, prophylaxie post-exposition; S.O., sans objet; VPH virus du papillome humain; VRZ, vaccin recombinant contre le zona; VVVCZ, vaccin à virus vivant contre le zona; $\geq$, supérieur ou égal à 


\section{Résumé et conclusion}

Le $\mathrm{GCl}$ continue de fournir des recommandations pratiques fondées sur des données probantes, formulées par le CCNI et le CCMTMV à l'intention des professionnels de la santé, afin d'éclairer les méthodes d'immunisation de première ligne. Le résumé des modifications se trouve périodiquement dans le Relevé des maladies transmissibles au Canada. On trouve également en ligne la liste des mises à jour des chapitres du $\mathrm{GCl}$ (18). Cette liste est mise à jour presque en temps réel. On peut également s'inscrire à la liste de distribution du CCNI et du $\mathrm{GCl}$ pour recevoir des avis faisant état des dernières recommandations du $\mathrm{CCNI}$, de ses déclarations, mises à jour et modifications aux chapitres du $\mathrm{GCl}$ (19).

\section{Déclaration des auteurs}

A. F. C. - Rédaction de la première ébauche, examen et révision M. T. - Examen et révision

A. H. - Examen et révision

\section{Conflit d'intérêts}

Aucuns.

\section{Remerciements}

Membres du CCNI : C Quach (présidente), W Vaudry (vice-présidente), N Dayneka, S Deeks, P DeWals, V Dubey, R Harrison, M Lavoie, M Salvadori, B Sander, C Rotstein, N Sicard, R Warrington

Représentants de liaison : J. Brophy (Association canadienne pour la recherche et l'évaluation en immunisation), E. Castillo (Société des obstétriciens et gynécologues du Canada), A. Cohn (Centers for Disease Control and Prevention, États-Unis), T. Cole (Comité canadien sur l'immunisation), J. Emili (Collège des médecins de famille du Canada), C. Mah (Association canadienne de santé publique), D. Moore (Société canadienne de pédiatrie), A. Pham-Huy (Association pour la microbiologie médicale et l'infectiologie Canada)

Représentants d'office : K Barnes (ministère de la Défense nationale et Forces armées canadiennes), G. Charos (Centre de I'immunisation et des maladies respiratoires infectieuses [CIMRI], Agence de la santé publique du Canada [ASPC]), J Gallivan (Direction des produits de santé commercialisés, Santé Canada [SC]); J Pennock (CIMRI, ASPC), R Pless (Direction des produits biologiques et des thérapies génétiques, SC), T Wong (Direction générale de la santé des Premières nations et des Inuits, SC)

\section{Financement}

Les travaux du CCNI sont financés par l'Agence de la santé publique du Canada.

\section{Références}

1. Desai S, Lsmail SJ, Lerch R, Warshawsky BF, Gemmill I. Canada's National Advisory Committee on Immunization: Celebrating 50 years. Can J Infect Dis Med Microbiol 2015 May-Jun;26(3):126-8. https://doi.org/10.1155/2015/431428 PubMed

2. Agence de la santé publique du Canada. Guide canadien d'immunisation : Introduction. Ottawa (ON): ASPC; 2017. https://www.canada.ca/fr/sante-publique/services/guidecanadien-immunisation/introduction.html

3. Agence de la santé publique du Canada. Guide canadien d'immunisation : Remerciements. Ottawa (ON): ASPC; 2016. https://www.canada.ca/fr/sante-publique/services/guidecanadien-immunisation/remerciements.html

4. Agence de la santé publique du Canada. Guide canadien d'immunisation: Partie 1 - Information clé sur I'immunisation; Méthodes d'administration des vaccins. Ottawa (ON): ASPC [mis à jour 2017]. https://www.canada. $\mathrm{ca} / \mathrm{fr} /$ sante-publique/services/publications/vie-saine/ guide-canadien-immunisation-partie-1-information-cleimmunisation/page-8-methodes-administration-vaccins.html

5. Agence de la santé publique du Canada. Guide canadien d'immunisation : Partie 3 - Vaccination de populations particulières; Immunisation des sujets immunodéprimés. Ottawa (ON): ASPC; 2018. https://www.canada.ca/fr/santepublique/services/publications/vie-saine/guide-canadienimmunisation-partie-3-vaccination-populations-particulieres/ page-8-immunisation-sujets-immunodeprimes.html

6. Agence de la santé publique du Canada. Immunisation durant la grossesse et l'allaitement: Guide canadien d'immunisation. Ottawa (ON): ASPC; [mis à jour 2018]. https://www.canada.ca/fr/sante-publique/services/ publications/vie-saine/guide-canadien-immunisationpartie-3-vaccination-populations-particulieres/page-4immunisation-durant-grossesse-allaitement.html

7. Agence de la santé publique du Canada. Vaccin contre le choléra et la diarrhée du voyageur due à Escherichia Coli entérotoxinogène (ECET). Ottawa (ON): ASPC; [mis à jour 2017]. https://www.canada.ca/fr/sante-publique/services/ publications/vie-saine/guide-canadien-immunisation-partie4-agents-immunisation-active/page-3-vaccin-contre-choleradiarrhee-voyageur-escherichia-coli-enterotoxinogene.html

8. Comité consultatif de la médecine tropicale et de la médecine des voyages. Déclaration sur la Diarrhée du Voyageur. Ottawa (ON); CCMTMV; [mis à jour 2015]. https:// www.canada.ca/fr/sante-publique/services/sante-voyageurs/ est-ccmtmv/declaration-diarrhee-voyageur.html

9. Agence de la santé publique du Canada. Chapitre sur la grippe du Guide canadien d'immunisation et Déclaration sur la vaccination antigrippale pour la saison 2018- 
2019. Ottawa (ON): ASPC; 2018. https://www.canada. $\mathrm{ca} / \mathrm{fr} /$ sante-publique/services/publications/vie-saine/ guide-canadien-immunisation-declaration-vaccinationantigrippale-2018-2019.html

10. Grifols Therapeutics Inc. Product monograph: GamaSTAN® S/D: Immune Globulin (Human), Solvent/Detergent Treated, Injectable Solution, 15-18\% Protein. 2018. https://pdf.hres. ca/dpd_pm/00043801.PDF

11. Agence de la santé publique du Canada. Guide canadien d'immunisation : Partie 4 - Agents d'immunisation active; Vaccin contre l'hépatite B. Ottawa (ON): ASPC; [mis à jour 2017]. https://www.canada.ca/fr/sante-publique/services/ publications/vie-saine/guide-canadien-immunisationpartie-4-agents-immunisation-active/page-7-vaccin-contrehepatite-b.html

12. Agence de la santé publique du Canada. Vaccin contre le zona: Guide canadien d'immunisation. ASPC; Ottawa (ON): ASPC; [mis à jour 2018]. https://www.canada.ca/fr/santepublique/services/publications/vie-saine/guide-canadienimmunisation-partie-4-agents-immunisation-active/page-8vaccin-contre-zona.html

13. Agence de la santé publique du Canada. Comité consultatif national de l'immunisation. Recommandations à jour sur I'utilisation des vaccins contre le zona. Ottawa (ON): ASPC; 2018. https://www.canada.ca/fr/services/sante/publications/ vie-saine/recommandations-jour-utilisation-vaccins-contrezona.html

14. Warrington $\mathrm{R}$, Ismail $\mathrm{S}$. Résumé de la mise à jour du CCNI sur les vaccins contre le zona. Relevé des maladies transmissibles au Canada 2018;44(9):249-554. DOI
15. Agence de la santé publique du Canada. Guide canadien d'immunisation : Partie 4 - Agents d'immunisation active; Vaccin contre le virus du papillome humain. Ottawa (ON): ASPC; [mis à jour 2018]. https://www.canada.ca/fr/santepublique/services/publications/vie-saine/guide-canadienimmunisation-partie-4-agents-immunisation-active/page-9vaccin-contre-virus-papillome-humain.html

16. Agence de la santé publique du Canada. Guide canadien d'immunisation: Partie 4 - Agents d'immunisation active; Vaccin contre la coqueluche. ASPC; Ottawa (ON): ASPC; [mis à jour 2018]. https://www.canada.ca/fr/santepublique/services/publications/vie-saine/guide-canadienimmunisation-partie-4-agents-immunisation-active/page-15vaccin-contre-coqueluche.html

17 Tunis MC, Salvadori MI, Dubey $\mathrm{V}$, Baclic $\mathrm{O}$ on behalf of the National Advisory Committee on Immunization (NACl). Updated NACI recommendations for measles post-exposure. Can Commun Dis Rep 2018;44(9):226-30. DOI

18. Agence de la santé publique du Canada. Guide canadien $d^{\prime}$ immunisation: Mises à jour des chapitres. Ottawa (ON): ASPC; 2018. https://www.canada.ca/fr/sante-publique/ services/guide-canadien-immunisation/mises-a-jourchapitres.html

19. Agence de la santé publique du Canada. Mises à jour du Guide canadien d'immunisation et publications du Comité consultatif national d'immunisation - Liste de diffusion. Ottawa (ON): ASPC; 2018. http://sante.canada.ca/fr/santecanada/services/healthy-living/immunization-and-vaccines/ canadian-immunization-guide/abonnement.html 